\title{
A POSTERIORI ERROR BOUNDS FOR PIECEWISE LINEAR APPROXIMATE SOLUTIONS OF ELLIPTIC EQUATIONS OF MONOTONE TYPE
}

\author{
KOICHI NIIJIMA
}

\begin{abstract}
We present a method for computing a posteriori error bounds for piecewise linear approximate solutions of elliptic equations of monotone type. The method is based on a relation between a line integral on an edge of a triangle and volume integrals in the triangle.
\end{abstract}

\section{INTRODUCTION}

There has been a great deal of work on a priori error estimates for numerical solutions of linear and nonlinear elliptic equations. A priori estimates can give convergence rates on mesh size, but cannot provide actual error bounds. A priori error bounds usually contain higher derivatives of exact solutions whose existence is often assumed.

In contrast with a priori error estimates, there are only a few results concerning a posteriori error estimates in essentially two-dimensional problems. Babuška and Rheinboldt have developed a theory of a posteriori error estimates for finite element solutions of one-dimensional linear problems [1]. Based on this theory, local mesh refinements have been carried out successfully for sample problems [3]. They have further extended their ideas to two-dimensional linear elliptic problems and have obtained lower and upper error estimators for finite element solutions [2]. However, in general, only approximations of these estimators can be computed. Bank and Weiser [4] have also given a method for getting a posteriori error bounds for finite element calculations. The problem treated there is a linear elliptic equation with a Neumann condition.

In this work, we propose a method for computing a posteriori error bounds for continuous piecewise linear approximate solutions of elliptic equations of monotone type. The fact that approximate solutions are piecewise linear plays an essential role in our analysis. Generally, numerical solutions do not necessarily have a continuous piecewise linear form. So the computed data are interpolated piecewise linearly in order to apply our method. We permit that the data include rounding errors. The success of our analysis lies in a relation between an integral on an edge of a triangle and volume integrals in the triangle. This relation will be given in Lemma 1 . Combining the relation with a jump in normal derivative of computed solutions at interelement boundaries enables

Received September 3, 1990; revised April 10, 1991.

1991 Mathematics Subject Classification. Primary 65N30. 
us to get an expression for an inner product on errors. The expression can be bounded from above using the Schwarz inequality. On the other hand, a bound from below of the inner product is easily derived with the aid of a monotonicity condition. Thus, computable error bounds can be obtained. The error bounds are quadratic functions of free parameters. Optimal error bounds are obtained by solving quadratic minimization problems. The feature of our method is that error estimates do not require the high regularity for the exact solution of the original problem. It suffices to assume only an $H^{1}$ regularity for the solution. In the last section, the effectiveness of our method will be examined through several numerical results.

\section{Problem AND NOTATION}

Let $\Omega$ be a bounded polyhedral domain in $R^{2}$ with boundary $\partial \Omega$. We consider the following problem:

$$
\begin{aligned}
-\Delta u+f(x, y, u, \nabla u) & =0 & & \text { in } \Omega, \\
u & =0 & & \text { on } \partial \Omega,
\end{aligned}
$$

where $\nabla u=\left(u_{x}, u_{y}\right)$.

Let $\omega$ be a subdomain in $\Omega$, and let $H^{1}(\omega)$ be the linear space of functions whose distributional first derivatives are in $L^{2}(\omega)$. By $H_{0}^{1}(\Omega)$ we denote the closure of $C_{0}^{\infty}(\Omega)$ in $H^{1}(\Omega)$. Denote the $L^{2}(\Omega)$ inner product by $(\cdot, \cdot)$, and the $L^{2}(\Omega)$ norm by $\|\cdot\|$.

A weak form of (2.1) and (2.2) is

$$
(\nabla u, \nabla v)+(f(x, y, u, \nabla u), v)=0
$$

for any $v \in H_{0}^{1}(\Omega)$.

We assume that

H1. The function $f$ satisfies the following growth condition:

$$
|f(x, y, s, t)| \leq C\left[z(x, y)+|s|^{\mu}+|t|^{\nu}\right]
$$

where $C$ is a positive constant, $z \in L^{p}(\Omega)$ with arbitrary $p>1$, and $\mu=p-1$, $\nu=2(p-1) / p$.

$\mathrm{H} 2$. There exist $\alpha \geq 0$ and $\beta>0$ such that for $v, w \in H_{0}^{1}(\Omega)$,

$$
\begin{aligned}
& (\nabla(v-w), \nabla(v-w))+(f(x, y, v, \nabla v)-f(x, y, w, \nabla w), v-w) \\
& \quad \geq \alpha\|v-w\|^{2}+\beta\|\nabla(v-w)\|^{2} .
\end{aligned}
$$

Under assumptions $\mathrm{H} 1$ and $\mathrm{H} 2,(2.3)$ has a unique solution in $H_{0}^{1}(\Omega)$ (see [5, pp. 143, 247]).

Consider a triangulation of $\Omega$ and denote the triangles by $\tau, \tau_{-}$, and $\tau_{+}$. Let $F$ be the set of triangles. The edges of triangles are denoted by $\gamma$ and $\gamma_{i}$. Let $E_{\Omega}$ be the set of edges not on $\partial \Omega$, and $E_{\partial \Omega}$ the set of edges on $\partial \Omega$. Put $E=E_{\Omega} \cup E_{\partial \Omega}$. The boundary of the triangle $\tau$ is denoted by $\partial \tau$. Let $(\cdot, \cdot)_{\tau}$ be the $L^{2}(\tau)$ inner product and $\langle\cdot, \cdot\rangle_{\gamma}$ the $L^{2}(\gamma)$ inner product.

For each edge in $E$, we determine a normal direction which is denoted by $n$. Let two triangles sharing an edge $\gamma$ in $E_{\Omega}$ be $\tau_{-}$and $\tau_{+}$, where $n$ is outward from $\tau_{-}$(see Figure 1). 


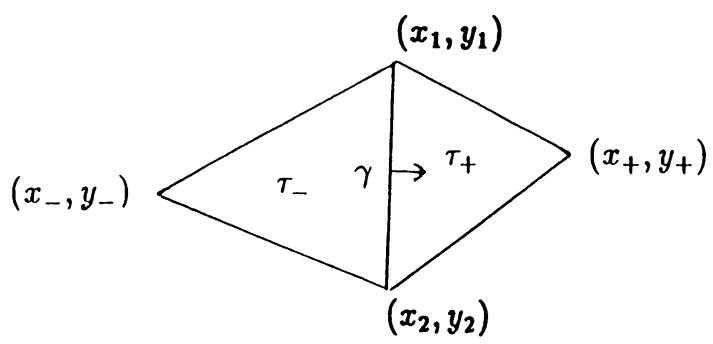

FIGURE 1

Let $h$ be the maximal diameter of the triangles and let $u^{h}$ be a continuous piecewise linear function. We define a jump in $\partial u^{h} / \partial n$ across $\gamma$ by

$$
\left[\frac{\partial u^{h}}{\partial n}\right]_{\gamma}=\left.\frac{\partial u^{h}}{\partial n}\right|_{\tau_{+}}-\left.\frac{\partial u^{h}}{\partial n}\right|_{\tau_{-}} .
$$

Note that this quantity does not depend on the direction of $n$. For later convenience, we define $\left[\frac{\partial u^{h}}{\partial n}\right]_{\gamma}=1$ for an edge $\gamma$ in $E_{\partial \Omega}$.

\section{A PRELIMINARY RESULT}

Let $\tau$ be a triangle and let the three edges of the triangle be $\gamma_{1}, \gamma_{2}$, and $\gamma_{3}$. Denote the vertices corresponding to $\gamma_{1}, \gamma_{2}$, and $\gamma_{3}$ by $\left(x_{1}, y_{1}\right),\left(x_{2}, y_{2}\right)$, and $\left(x_{3}, y_{3}\right)$, respectively.

We have the following lemma.

Lemma 1. For $g \in H^{1}(\tau)$, it holds that

$$
\langle 1, g\rangle_{\gamma_{3}}=\frac{\left|\gamma_{3}\right|}{\left|J_{\tau}\right|}\left[2(1, g)_{\tau}+\left(x-x_{3}, g_{x}\right)_{\tau}+\left(y-y_{3}, g_{y}\right)_{\tau}\right] \text {, }
$$

where

$$
J_{\tau}=\left(x_{2}-x_{1}\right)\left(y_{3}-y_{1}\right)-\left(x_{3}-x_{1}\right)\left(y_{2}-y_{1}\right)
$$

and $\left|\gamma_{3}\right|$ indicates the length of $\gamma_{3}$.

Proof. Let $T$ be a triangle having the vertices $O(0,0), P(1,0)$, and $Q(0,1)$, and give the function $G \in H^{1}(T)$. First we show that

$$
\begin{aligned}
\frac{1}{2} \int_{0}^{1} G(X, 0) d X= & \int_{T} G(X, Y) d X d Y+\frac{1}{2} \int_{T} X \frac{\partial G}{\partial X}(X, Y) d X d Y \\
& -\frac{1}{2} \int_{T}(1-Y) \frac{\partial G}{\partial Y}(X, Y) d X d Y
\end{aligned}
$$

Integrating by parts,

$$
\begin{aligned}
\int_{T} X & \frac{\partial G}{\partial X}(X, Y) d X d Y=\int_{0}^{1}\left[\int_{0}^{1-Y} X \frac{\partial G}{\partial X}(X, Y) d X\right] d Y \\
& =\int_{0}^{1}\left[(1-Y) G(1-Y, Y)-\int_{0}^{1-Y} G(X, Y) d X\right] d Y \\
& =\int_{0}^{1}(1-Y) G(1-Y, Y) d Y-\int_{T} G(X, Y) d X d Y
\end{aligned}
$$




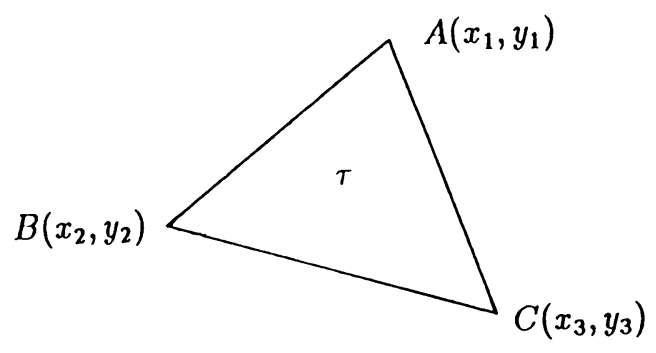

FIGURE 2

Using again integration by parts, we get

$$
\begin{aligned}
\int_{T}(1 & -Y) \frac{\partial G}{\partial Y}(X, Y) d X d Y=\int_{0}^{1}\left[\int_{0}^{1-X}(1-Y) \frac{\partial G}{\partial Y}(X, Y) d Y\right] d X \\
& =\int_{0}^{1}\left[X G(X, 1-X)-G(X, 0)+\int_{0}^{1-X} G(X, Y) d Y\right] d X \\
& =\int_{0}^{1} X G(X, 1-X) d X-\int_{0}^{1} G(X, 0) d X+\int_{T} G(X, Y) d X d Y .
\end{aligned}
$$

Since

$$
\int_{0}^{1}(1-Y) G(1-Y, Y) d Y=\int_{0}^{1} X G(X, 1-X) d X,
$$

substituting (3.2) and (3.3) into the right-hand side of (3.1) leads to the left-hand side of (3.1).

We shall derive the formula in Lemma 1. Consider the triangle $\tau$ with the vertices $A\left(x_{1}, y_{1}\right), B\left(x_{2}, y_{2}\right)$, and $C\left(x_{3}, y_{3}\right)$ (see Figure 2).

A linear transformation mapping $O, P$, and $Q$ in the master element to $A, B$, and $C$, respectively, is given by

$$
\begin{aligned}
& x=\left(x_{2}-x_{1}\right) X+\left(x_{3}-x_{1}\right) Y+x_{1}, \\
& y=\left(y_{2}-y_{1}\right) X+\left(y_{3}-y_{1}\right) Y+y_{1} .
\end{aligned}
$$

For $g \in H^{1}(\tau)$, we define $G(X, Y)$ by

$$
G(X, Y) \equiv g\left(\left(x_{2}-x_{1}\right) X+\left(x_{3}-x_{1}\right) Y+x_{1},\left(y_{2}-y_{1}\right) X+\left(y_{3}-y_{1}\right) Y+y_{1}\right)
$$

and apply formula (3.1) to this $G$. By the change of variables $h_{3} X=s$ with $h_{3}=\sqrt{\left(x_{2}-x_{1}\right)^{2}+\left(y_{2}-y_{1}\right)^{2}}$, the left-hand side of $(3.1)$ becomes

$$
\frac{1}{2} \int_{0}^{1} G(X, 0) d X=\frac{1}{2 h_{3}} \int_{0}^{h_{3}} g\left(\frac{x_{2}-x_{1}}{h_{3}} s+x_{1}, \frac{y_{2}-y_{1}}{h_{3}} s+y_{1}\right) d s .
$$

On the other hand, since the Jacobian takes the form

$$
J_{\tau}=\left(x_{2}-x_{1}\right)\left(y_{3}-y_{1}\right)-\left(x_{3}-x_{1}\right)\left(y_{2}-y_{1}\right)
$$

and $d X d Y=\left|J_{\tau}\right| d x d y$, we have

$$
\int_{T} G(X, Y) d X d Y=\frac{1}{\left|J_{\tau}\right|} \int_{\tau} g(x, y) d x d y .
$$


Using the relations

$$
\begin{aligned}
& X=\frac{1}{J_{\tau}}\left[\left(x-x_{1}\right)\left(y_{3}-y_{1}\right)-\left(x_{3}-x_{1}\right)\left(y-y_{1}\right)\right], \\
& Y=\frac{1}{J_{\tau}}\left[\left(x_{2}-x_{1}\right)\left(y-y_{1}\right)-\left(x-x_{1}\right)\left(y_{2}-y_{1}\right)\right],
\end{aligned}
$$

we further have

$$
\begin{aligned}
& \frac{\partial G}{\partial X}(X, Y)=\frac{\partial g(x, y)}{\partial x}\left(x_{2}-x_{1}\right)+\frac{\partial g(x, y)}{\partial y}\left(y_{2}-y_{1}\right) \\
& \frac{\partial G}{\partial Y}(X, Y)=\frac{\partial g(x, y)}{\partial x}\left(x_{3}-x_{1}\right)+\frac{\partial g(x, y)}{\partial y}\left(y_{3}-y_{1}\right)
\end{aligned}
$$

Consequently,

$$
\begin{aligned}
\int_{T} X & \frac{\partial G}{\partial X}(X, Y) d X d Y \\
= & \frac{1}{\left|J_{\tau}\right| J_{\tau}} \int_{\tau}\left[\left(x-x_{1}\right)\left(y_{3}-y_{1}\right)-\left(x_{3}-x_{1}\right)\left(y-y_{1}\right)\right] \\
\cdot & {\left[\frac{\partial g(x, y)}{\partial x}\left(x_{2}-x_{1}\right)+\frac{\partial g(x, y)}{\partial y}\left(y_{2}-y_{1}\right)\right] d x d y }
\end{aligned}
$$

Substituting (3.4), (3.5), (3.6), and (3.7) into (3.1) yields

$$
\begin{aligned}
\int_{0}^{h_{3}} g & \left(\frac{x_{2}-x_{1}}{h_{3}} s+x_{1}, \frac{y_{2}-y_{1}}{h_{3}} s+y_{1}\right) d s \\
= & \frac{2 h_{3}}{\left|J_{\tau}\right|} \int_{\tau} g(x, y) d x d y+\frac{h_{3}}{\left|J_{\tau}\right|} \int_{\tau}\left(x-x_{3}\right) \frac{\partial g(x, y)}{\partial x} d x d y \\
& +\frac{h_{3}}{\left|J_{\tau}\right|} \int_{\tau}\left(y-y_{3}\right) \frac{\partial g(x, y)}{\partial y} d x d y
\end{aligned}
$$

whose inner product version is the desired result.

This relation is a formula changing a line integral on an edge into the sum of three volume integrals over $\tau$. By virtue of this formula, we can rewrite the line integrals appearing in partial integrations of the gradient term by elementwise volume integrals.

\section{Main Results}

Let $u^{h}$ be a continuous piecewise linear interpolant for data obtained by solving (2.3) by some approximate methods. From now on, we call this $u^{h}$ a piecewise linear interpolate solution of (2.3). Let $\tau$ be any triangle in $F$ and denote the three edges of $\tau$ by $\gamma_{1}, \gamma_{2}$, and $\gamma_{3}$. 
We now define an operator $\Delta_{\tau}^{h}$ by

$$
\Delta_{\tau}^{h} u^{h}=\frac{2}{\left|J_{\tau}\right|} \sum_{i=1}^{3} w_{\tau, i}\left|\gamma_{i}\right|\left[\frac{\partial u^{h}}{\partial n}\right]_{\gamma_{i}} .
$$

Here, if $\gamma_{i}$ is in $E_{\Omega}$, then the corresponding $w_{\tau, i}$ denotes a free parameter satisfying

$$
w_{\tau, i}+w_{\tau^{\prime}, i^{\prime}}=1
$$

for the parameter $w_{\tau^{\prime}, i^{\prime}}$, where $\tau^{\prime}$ indicates the other triangle sharing $\gamma_{i}$. If $\gamma_{i}$ is in $E_{\partial \Omega}$, then the corresponding $w_{\tau, i}$ is free and $\left[\frac{\partial u^{h}}{\partial n}\right]_{\gamma_{i}}=1$ as defined before.

Using the same symbols as above, we define a two-dimensional vector $r_{\tau}^{h}$ as follows:

$$
r_{\tau}^{h}=\frac{1}{\left|J_{\tau}\right|}\left(\sum_{i=1}^{3} w_{\tau, i}\left|\gamma_{i}\right|\left[\frac{\partial u^{h}}{\partial n}\right]_{\gamma_{i}}\left(x-x_{i}\right), \sum_{i=1}^{3} w_{\tau, i}\left|\gamma_{i}\right|\left[\frac{\partial u^{h}}{\partial n}\right]_{\gamma_{i}}\left(y-y_{i}\right)\right) .
$$

We define $\Delta^{h}$ and $r^{h}$ by $\Delta^{h}=\left(\Delta_{\tau}^{h}\right)_{\tau \in F}$ and $r^{h}=\left(r_{\tau}^{h}\right)_{\tau \in F}$, respectively. By $W$, we denote the set of vectors whose components consist of all $w_{\tau}$.

The following lemma holds.

Lemma 2. Let $u$ be a solution of (2.3) and let $u^{h}$ be a piecewise linear interpolate solution of (2.3). We put $e=u-u^{h}$ and define $L$ by

$$
L=(\nabla e, \nabla e)+\left(f(x, y, u, \nabla u)-f\left(x, y, u^{h}, \nabla u^{h}\right), e\right) .
$$

Then we have

$$
L=-\left(-\Delta^{h} u^{h}+f^{h}, e\right)+\left(r^{h}, \nabla e\right),
$$

where we set $f^{h}=f\left(x, y, u^{h}, \nabla u^{h}\right)$ for simplicity.

Proof. By the definition of $e$,

$$
L=(\nabla u, \nabla e)+(f(x, y, u, \nabla u), e)-\left(\nabla u^{h}, \nabla e\right)-\left(f\left(x, y, u^{h}, \nabla u^{h}\right), e\right) .
$$

Choosing $v=e$ in (2.3), we get

$$
(\nabla u, \nabla e)+(f(x, y, u, \nabla u), e)=0 .
$$

Hence,

$$
L=-\left(\nabla u^{h}, \nabla e\right)-\left(f\left(x, y, u^{h}, \nabla u^{h}\right), e\right) .
$$

We put

$$
M=-\left(\nabla u^{h}, \nabla e\right)
$$

and rewrite $M$ as

$$
M=-\sum_{\tau \in F}\left(\nabla u^{h}, \nabla e\right)_{\tau} .
$$

Integrating each term by parts yields

$$
M=-\sum_{\tau \in F}\left\langle\frac{\partial u^{h}}{\partial n_{0}}, e\right\rangle_{\partial \tau},
$$


where $n_{0}$ is the outward normal for $\tau$. Noticing that the outward normal for $\tau$ is the inward normal for a neighbor sharing a common edge, we may write $M$ as

$$
M=\sum_{\gamma \in E_{\Omega}}\left\langle\left[\frac{\partial u^{h}}{\partial n}\right]_{\gamma}, e\right\rangle_{\gamma}-\sum_{\gamma \in E_{\partial \Omega}}\left\langle\frac{\partial u^{h}}{\partial n_{0}}, e\right\rangle_{\gamma} .
$$

For the first term of the right-hand side, we remark that $\left[\frac{\partial u^{h}}{\partial n}\right]_{\gamma}$ is constant on $\gamma \in E_{\Omega}$. For the second term of the right-hand side, we can write a free parameter $-w_{\tau}$ instead of $\frac{\partial u^{h}}{\partial n_{0}}, \tau$ being a triangle with the edge $\gamma$, since $\left.e\right|_{\gamma}=0$ for $\gamma$ in $E_{\partial \Omega}$. Thus we have

$$
M=\sum_{\gamma \in E_{\Omega}}\left[\frac{\partial u^{h}}{\partial n}\right]_{\gamma}\langle 1, e\rangle_{\gamma}+\sum_{\gamma \in E_{\partial \Omega}} w_{\tau}\langle 1, e\rangle_{\gamma} .
$$

For an edge $\gamma$ in $E_{\Omega}$, let two triangles sharing $\gamma$ be $\tau_{-}$and $\tau_{+}$as in Figure 1. By Lemma 1,

$$
\begin{aligned}
& \langle 1, e\rangle_{\gamma}=\frac{|\gamma|}{\left|J_{-}\right|}\left[2(1, e)_{\tau_{-}}+\left(x-x_{-}, e_{x}\right)_{\tau_{-}}+\left(y-y_{-}, e_{y}\right)_{\tau_{-}}\right], \\
& \langle 1, e\rangle_{\gamma}=\frac{|\gamma|}{\left|J_{+}\right|}\left[2(1, e)_{\tau_{+}}+\left(x-x_{+}, e_{x}\right)_{\tau_{+}}+\left(y-y_{+}, e_{y}\right)_{\tau_{+}}\right],
\end{aligned}
$$

where

$$
\begin{aligned}
& J_{-}=\left(x_{2}-x_{1}\right)\left(y_{-}-y_{1}\right)-\left(x_{-}-x_{1}\right)\left(y_{2}-y_{1}\right), \\
& J_{+}=\left(x_{2}-x_{1}\right)\left(y_{+}-y_{1}\right)-\left(x_{+}-x_{1}\right)\left(y_{2}-y_{1}\right) .
\end{aligned}
$$

Multiplying both sides of $(4.3)$ and $(4.4)$ by $w_{\tau_{-}}$and $w_{\tau_{+}}$, which satisfy $w_{\tau_{-}}+$ $w_{\tau_{+}}=1$, and summing the resulting equations, we get

$$
\begin{aligned}
\langle 1, e\rangle_{\gamma}= & 2\left[w_{\tau_{-}} \frac{|\gamma|}{\left|J_{-}\right|}(1, e)_{\tau_{-}}+w_{\tau_{+}} \frac{|\gamma|}{\left|J_{+}\right|}(1, e)_{\tau_{+}}\right] \\
& +w_{\tau_{-}} \frac{|\gamma|}{\left|J_{-}\right|}\left[\left(x-x_{-}, e_{x}\right)_{\tau_{-}}+\left(y-y_{-}, e_{y}\right)_{\tau_{-}}\right] \\
& +w_{\tau_{+}} \frac{|\gamma|}{\left|J_{+}\right|}\left[\left(x-x_{+}, e_{x}\right)_{\tau_{+}}+\left(y-y_{+}, e_{y}\right)_{\tau_{+}}\right] .
\end{aligned}
$$

For an edge $\gamma$ in $E_{\partial \Omega}$, we adopt (4.3) or (4.4). Substituting these expressions into (4.2) and changing the indexes properly, we have, by the definition of $\Delta^{h}$ and $r^{h}$,

$$
M=\left(\Delta^{h} u^{h}, e\right)+\left(r^{h}, \nabla e\right)
$$

We further have by $(4.1)$,

$$
L=-\left(-\Delta^{h} u^{h}+f^{h}, e\right)+\left(r^{h}, \nabla e\right),
$$

which proves Lemma 2.

We are now ready to describe our main result.

Theorem. Let $u$ be a solution of (2.3) and let $u^{h}$ be a piecewise linear interpolate solution of (2.3). Let $\alpha$ and $\beta$ be defined in assumption $\mathrm{H} 2$. Then we have, for $e=u-u^{h}$,

$$
(2 \alpha-\rho)\|e\|^{2}+(2 \beta-\lambda)\|\nabla e\|^{2} \leq \inf _{W}\left(\frac{1}{\rho}\left\|-\Delta^{h} u^{h}+f^{h}\right\|^{2}+\frac{1}{\lambda}\left\|r^{h}\right\|^{2}\right)
$$


for $\alpha>0$, where $\rho$ and $\lambda$ satisfy $2 \alpha \geq \rho>0$ and $2 \beta \geq \lambda>0$, and

$$
2 \alpha\|e\|^{2}+\beta\|\nabla e\|^{2} \leq \frac{1}{\beta} \inf _{W}\left(\frac{1}{K}\left\|-\Delta^{h} u^{h}+f^{h}\right\|+\left\|r^{h}\right\|\right)^{2}
$$

for $\alpha \geq 0$, in which

$$
K=\pi \sqrt{\frac{1}{l_{x}^{2}}+\frac{1}{l_{y}^{2}}},
$$

where $l_{x}$ and $l_{y}$ indicate the length of the projections of $\Omega$ into the $x$-axis and $y$-axis, respectively.

Proof. By assumption $\mathrm{H} 2$, we have for $L$ in Lemma 2,

$$
L \geq \alpha\|e\|^{2}+\beta\|\nabla e\|^{2} .
$$

On the other hand, Lemma 2 gives

$$
\begin{aligned}
L & \leq\left|\left(-\Delta^{h} u^{h}+f^{h}, e\right)\right|+\left|\left(r^{h}, \nabla e\right)\right| \\
& \leq\left\|-\Delta^{h} u^{h}+f^{h}\right\|\|e\|+\left\|r^{h}\right\|\|\nabla e\| .
\end{aligned}
$$

Hence,

$$
\alpha\|e\|^{2}+\beta\|\nabla e\|^{2} \leq\left\|-\Delta^{h} u^{h}+f^{h}\right\|\|e\|+\left\|r^{h}\right\|\|\nabla e\| .
$$

Applying the Schwarz inequality to the right-hand side of (4.5), we get

$$
\begin{aligned}
& \left\|-\Delta^{h} u^{h}+f^{h}\right\|\|e\|+\left\|r^{h}\right\|\|\nabla e\| \\
& \quad \leq \frac{1}{2}\left(\rho\|e\|^{2}+\lambda\|\nabla e\|^{2}\right)+\frac{1}{2}\left(\frac{1}{\rho}\left\|-\Delta^{h} u^{h}+f^{h}\right\|^{2}+\frac{1}{\lambda}\left\|r^{h}\right\|^{2}\right) .
\end{aligned}
$$

Combining this with (4.5) and taking the inf of $\frac{1}{\rho}\left\|-\Delta^{h} u^{h}+f^{h}\right\|^{2}+\frac{1}{\lambda}\left\|r^{h}\right\|^{2}$ proves the first estimate.

The second estimate is derived as follows: By the Poincare inequality, $\|e\| \leq$ $\|\nabla e\| / K[6$, p. 196$]$ and by Schwarz's inequality,

$$
\begin{aligned}
&\left\|-\Delta^{h} u^{h}+f^{h}\right\|\|e\|+\left\|r^{h}\right\|\|\nabla e\| \\
& \leq\left(\frac{1}{K}\left\|-\Delta^{h} u^{h}+f^{h}\right\|+\left\|r^{h}\right\|\right)\|\nabla e\| \\
& \leq \frac{\beta}{2}\|\nabla e\|^{2}+\frac{1}{2 \beta}\left(\frac{1}{K}\left\|-\Delta^{h} u^{h}+f^{h}\right\|+\left\|r^{h}\right\|\right)^{2} .
\end{aligned}
$$

Combining this with (4.5) and taking the inf of $\left(\frac{1}{K}\left\|-\Delta^{h} u^{h}+f^{h}\right\|+\left\|r^{h}\right\|\right)^{2}$ gives the second estimate.

\section{NUMERICAL RESULTS}

In this section, we apply our a posteriori error estimating method to three nonlinear elliptic problems and verify the effectiveness of our method. 


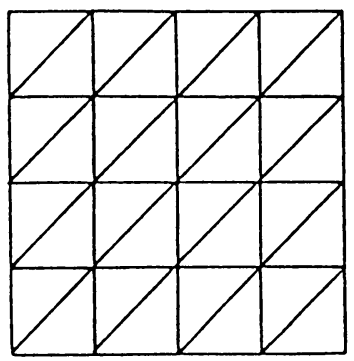

FIGURE 3

The first problem is

\section{Problem 1.}

$$
\begin{aligned}
-\Delta u+u^{3}+u & =g_{1} \quad \text { in } \Omega=(0,1) \times(0,1), \\
\left.u\right|_{\partial \Omega} & =0,
\end{aligned}
$$

where $g_{1}$ is chosen such that

$$
u(x, y)=8 x(y-1) \sin (\pi y(x-1))
$$

satisfies the above equation. It is easy to verify assumption $\mathrm{H} 1$. Assumption $\mathrm{H} 2$ is fulfilled as follows:

$$
\begin{aligned}
& (\nabla(v-w), \nabla(v-w))+\left(v^{3}+v-w^{3}-w, v-w\right) \\
& \quad \geq\|v-w\|^{2}+\|\nabla(v-w)\|^{2}
\end{aligned}
$$

because $\left(v^{3}-w^{3}, v-w\right) \geq 0$ holds. Therefore, the two estimates in our theorem can be applied with $\alpha=\beta=1$. For the present problem, we use the second estimate, since it gives sharper bounds than the first.

The interval $(0,1)$ is divided into $m$ equidistant subintervals and a triangulation is made as in Figure 3.

Numerical solutions $u^{h}$ with a piecewise linear form were obtained by minimizing the functional

$$
\int_{\Omega}\left[\frac{1}{2}\left|\nabla u^{h}\right|^{2}+\frac{1}{4}\left(u^{h}\right)^{4}+\frac{1}{2}\left(u^{h}\right)^{2}-g_{1} u^{h}\right] d x d y
$$

Next, optimal error bounds were computed by solving the quadratic minimization problem

$$
\inf _{W}\left[\frac{1}{K^{2}}\left\|-\Delta^{h} u^{h}+\left(u^{h}\right)^{3}+u^{h}-g_{1}\right\|^{2}+\left\|r^{h}\right\|^{2}\right]
$$

where $K=\sqrt{2} \pi$. We compared these optimal error bounds with the exact errors $\sqrt{2\|e\|^{2}+\|\nabla e\|^{2}}$. A posteriori error bounds using natural parameters were also computed for comparison. 


$\begin{array}{rccc}m & \sqrt{2\|e\|^{2}+\|\nabla e\|^{2}} & \text { optimal error bounds } & \begin{array}{c}\text { error bounds in the case that } \\ \text { all parameters are equal to } 0.5\end{array} \\ 4 & 1.462 & 2.448 & 4.446 \\ 6 & 0.998 & 1.671 & 3.396 \\ 8 & 0.745 & 1.263 & 2.851 \\ 10 & 0.597 & 1.014 & 2.532 \\ 12 & 0.498 & 0.847 & 2.328 \\ 14 & 0.427 & 0.727 & 2.189 \\ 16 & 0.374 & 0.636 & 2.087\end{array}$

We see from this table that the ratio of the optimal error bounds to the exact errors is about 1.7. The error bounds using natural parameters are too large.

The second problem is

\section{Problem 2.}

$$
\begin{aligned}
-\Delta u+u^{3} & =g_{2} \quad \text { in } \Omega=(0,1) \times(0,1), \\
\left.u\right|_{\partial \Omega} & =0 .
\end{aligned}
$$

The function $g_{2}$ is chosen such that

$$
u(x, y)=8 x(y-1) \sin (\pi y(x-1))
$$

satisfies the above equation. Assumption $\mathrm{H} 1$ is easily verified. Assumption $\mathrm{H} 2$ is fulfilled with $\alpha=0$ and $\beta=1$, since

$$
(\nabla(v-w), \nabla(v-w))+\left(v^{3}-w^{3}, v-w\right) \geq\|\nabla(v-w)\|^{2} .
$$

Hence, the second estimate in our theorem holds. We make a triangulation as in Problem 1 (see Figure 3). Piecewise linear solutions $u^{h}$ were computed by minimizing the functional

$$
\int_{\Omega}\left[\frac{1}{2}\left|\nabla u^{h}\right|^{2}+\frac{1}{4}\left(u^{h}\right)^{4}-g_{2} u^{h}\right] d x d y .
$$

Optimal error bounds were obtained by solving the quadratic minimization problem

$$
\inf _{W}\left[\frac{1}{K^{2}}\left\|-\Delta^{h} u^{h}+\left(u^{h}\right)^{3}-g_{2}\right\|^{2}+\left\|r^{h}\right\|^{2}\right],
$$

where $K=\sqrt{2} \pi$. As in Problem 1, we compared such optimal errors with the exact errors $\|\nabla e\|$ and a posteriori errors using natural parameters.

$\begin{array}{rrcc}m & \|\nabla e\| & \text { optimal error bounds } & \begin{array}{c}\text { error bounds in the case that } \\ \text { all parameters are equal to } 0.5\end{array} \\ 4 & 1.455 & 2.440 & 4.432 \\ 6 & 0.986 & 1.668 & 3.391 \\ 8 & 0.744 & 1.262 & 2.848 \\ 10 & 0.597 & 1.014 & 2.531 \\ 12 & 0.498 & 0.847 & 2.328 \\ 14 & 0.427 & 0.727 & 2.188 \\ 16 & 0.374 & 0.636 & 2.086\end{array}$


The data in this table are almost the same as in the first table.

The last problem is

\section{Problem 3.}

$$
\begin{aligned}
-\Delta u+\cos (u) & =g_{3} \quad \text { in } \Omega=(0,1) \times(0,1), \\
\left.u\right|_{\partial \Omega} & =0 .
\end{aligned}
$$

The function $g_{3}$ is chosen such that

$$
u(x, y)=8 x(y-1) \sin (\pi y(x-1))
$$

satisfies the above equation. Assumption $\mathrm{H} 1$ is easily verified. Since

$$
\begin{aligned}
& (\nabla(v-w), \nabla(v-w))+(\cos (v)-\cos (w), v-w) \\
& \quad \geq\|\nabla(v-w)\|^{2}-\|v-w\|^{2} \geq\left(1-\frac{1}{K^{2}}\right)\|\nabla(v-w)\|^{2}
\end{aligned}
$$

for $K=\sqrt{2} \pi$, assumption $\mathrm{H} 2$ is fulfilled with $\alpha=0$ and $\beta=1-1 / K^{2}$. Thus the second estimate in our theorem holds. We make a triangulation as in Problem 1 (see Figure 3). Piecewise linear solutions $u^{h}$ were obtained by minimizing the functional

$$
\int_{\Omega}\left[\frac{1}{2}\left|\nabla u^{h}\right|^{2}+\sin \left(u^{h}\right)-g_{3} u^{h}\right] d x d y
$$

The quadratic minimization problem

$$
\inf _{W}\left[\frac{1}{K^{2}}\left\|-\Delta^{h} u^{h}+\cos \left(u^{h}\right)-g_{3}\right\|^{2}+\left\|r^{h}\right\|^{2}\right]
$$

was solved to get optimal error bounds. Furthermore, these optimal errors were compared with the exact errors $\|\nabla e\|$ and a posteriori errors using natural parameters.

$\begin{array}{rrcc}m & \|\nabla e\| & \text { optimal error bounds } & \begin{array}{c}\text { error bounds in the case that } \\ \text { all parameters are equal to } 0.5\end{array} \\ 4 & 1.458 & 2.557 & 4.658 \\ 6 & 0.987 & 1.753 & 3.569 \\ 8 & 0.744 & 1.327 & 2.999 \\ 10 & 0.597 & 1.067 & 2.665 \\ 12 & 0.498 & 0.891 & 2.452 \\ 14 & 0.427 & 0.765 & 2.305 \\ 16 & 0.374 & 0.670 & 2.198\end{array}$

The ratio of the optimal errors to the exact errors is about 1.8. The results obtained using natural parameters are too large.

The experiment was performed by using Turbo Pascal Ver.5.5 on the personal computer EPSON PC-286UX. 


\section{BIBLIOGRAPHY}

1. I. Babuška and W. C. Rheinboldt, A-posteriori error estimates for the finite element method, Internat. J. Numer. Methods Engrg. 12 (1978), 1597-1615.

2. _ Error estimates for adaptive finite element computations, SIAM J. Numer. Anal. 15 (1978), 736-754.

3. __ Analysis of optimal finite-element meshes in $R^{1}$, Math. Comp. 33 (1979), 435-463.

4. R. E. Bank and A. Weiser, Some a posteriori error estimators for elliptic partial differential equations, Math. Comp. 44 (1985), 283-301.

5. S. Fučík and A. Kufner, Nonlinear differential equations, Elsevier, Amsterdam, 1980.

6. K. Rektorys, Variational methods in mathematics, science and engineering, Reidel, Holland, 1980.

Department of Control Engineering and Science, Kyushu Institute of Technology, IIZUKA 820, JAPAN

E-mail address: niijima@ces.kyutech.ac.jp 